\title{
ORIGINAL
}

\section{ADAPTACIÓN AL ESPAÑOL DEL CUESTIONARIO VECÚ ET SANTE PERÇUE DE L'ADOLESCENT (VSP-A): UNA MEDIDA GENÉRICA DE CALIDAD DE VIDA PARA ADOLESCENTES (*)}

Vicky Serra-Sutton (1), Michael Herdman (2), Luis Rajmil (1), Rosalía Santed (3), Montse Ferrer (3), Marie Claude Siméoni (4) y Pascal Auquier (4)

(1) Agència d'Avaluació de Tecnologia i Recerca Mèdiques, Barcelona

(2) 3D Health Research, Barcelona

(3) Institut Municipal d'Investigació Mèdica, Barcelona

(4) Departamento de Salud Pública, Facultad de Medicina, Marsella, Francia

(*) Estudio financiando por el Observatoire Regional d'Epidemiologie de la Region Provence-Alpes-Cote d'Azur, Faculté de Medicine, Marseille

\section{RESUMEN}

Fundamentos: En la última década se han desarrollado diversas medidas de calidad de vida relacionada con la salud para uso exclusivo en niños/as y adolescentes. No obstante, existen pocos instrumentos de estas características adaptados en España. El Vecú et Sante Perçue de l'adolescent (VSP-A) es un instrumento genérico de calidad de vida relacionada con la salud para adolescentes de 11 a 17 años desarrollado en Francia. E objetivo de este estudio fue adaptar al español el VSP-A, como primera fase para la obtención del cuestionario.

Métodos: Se adaptó la versión del VSP-A de 39 preguntas siguiendo la metodología de traducción directa e inversa incluyendo: 2 traducciones al español, puntuación del grado de dificultad (0 min-10 máx) y clasificación de equivalencia semántica y cultural, 2 reuniones y discusión en paneles de adolescentes, as como reuniones de consenso del equipo de investigación. Finalmente se realizó una traducción inversa (retro-traducción) al francés y se administró la versión final pre-test en la prueba piloto.

Resultados: La mayoría de las preguntas se clasificaron como equivalentes (24 sobre 39$)$. Tras las reuniones con adolescentes se modificaron algunas preguntas. Tras la retro-traducción, 3 preguntas necesitaron cambios menores.

Conclusiones: La versión española del VSP-A parece semántica y culturalmente equivalente a la versión original en francés y adecuada para adolescentes en España. La sencillez de las preguntas, los comentarios de los adolescentes y la participación de los autores originales en el proceso de adaptación ha permitido obtener una versión pre-test adecuada. La siguiente fase del estudio es la comprobación de la fiabilidad y validez. Se espera que el VSP-A sea de utilidad para medir la calidad de vida relacionada con la salud en encuestas de salud o como instrumento de cribado en colegios o centros de atención primaria en nuestro medio.

Palabras clave: Adolescencia. Calidad de vida. Cuestionario. España. Comparación transcultural

Correspondencia:

Luis Rajmil

Agència d'Avaluació de Tecnologia i Recerca Mèdiques

Trav de les Corts 131-159

Pavelló Ave Maria.

08028 Barcelona

Correo electrónico: 1rajmil@catsalut.net

\section{ABSTRACT}

\section{Cross-cultural Adaptation into Spanish of the Vecú et Sante Perçue de l'Adolescent (VSP-A) into Spanish: a Generic Measure of Quality of Life for Adolescents}

Background: Different measures of health related quality of life for use exclusively in children and adolescents have been developed over the last ten years. However, few instruments of this type have been adapted in Spain. The VSP-A is a generic health related quality of life measure for adolescents aged 11-17 developed in France. The objective of this study was to adapt the VSP-A into Spanish as a first step towards obtaining this questionnaire.

Methods: The version of the VSP-A including 39 questions was adapted following the foward-backward translation methodology, including two translations into Spanish, scoring of difficulty ( 0 min-10 max.) and classification of semantic and conceptual equivalence, two panel discussions with adolescents, as well as meetings of consensus with the original authors. Finally, a backward translation (translation back into the original language) was carried out into French, and the final pre-test version was administered in a pilot test.

Results: Most of the questions were classified as equivalent (24/39). Following the meetings with adolescents, changes were made in some questions. After the back-translation into French, three questions required some minor changes.

Conclusions: The Spanish version of the VSP-A seems to be semantically and culturally equivalent to the original version and suitable for adolescents in Spain. The inclusion of easy statements in the original version, the comments of the adolescents and the involvement in the adaptation process of the original authors has offered the possibility of achieving a suitable pre-test version. The next phase of the study will involve the questionnaire's reliability and validity testing. The VSP-A is expected to be useful for measuring the health-related quality of life in health surveys or as a screening tool in schools and primary care centers in Spain.

Key words: Adolescence. Quality of Life. Questionnaires. Health surveys. Cognitive debrifing. Cross-cultural comparison. 


\section{INTRODUCCIÓN}

Existe un interés creciente en medir la calidad de vida relacionada con la salud (CVRS), tanto en la investigación y en la práctica clínica, como en la evaluación de servicios sanitarios y en salud pública ${ }^{1-5}$. Los instrumentos de medida de CVRS recogen la valoración subjetiva del individuo respecto de su estado de salud y su bienestar general y la influencia de éstos sobre la capacidad para llevar a cabo las actividades consideradas importantes por el propio individuo $^{6}$. Las dimensiones que habitualmente recogen los instrumentos de CVRS son: el funcionamiento físico, psicológico y social; la movilidad y el cuidado personal; y el bienestar emocional ${ }^{7}$. La mayoría de instrumentos de CVRS han sido diseñados para los adultos, si bien en la última década se han desarrollado diversas medidas para uso exclusivo en niños/as y adolescentes ${ }^{8}$, aunque en España existen pocos ${ }^{9}$.

El Vecú et Santé Perçue de l'Adolescent (VSP-A) es un instrumento genérico de CVRS, autoadministrado, para adolescentes de 11 a 17 años, que ha sido desarrollado en la Universidad de Marsella (Francia) ${ }^{10,11}$. Este cuestionario fue creado a partir de la opinión de los adolescentes sobre lo que constituía lo más importante sobre su salud percibida. Inicialmente se generaron 118 preguntas a partir de 73 entrevistas en profundidad con adolescentes que recogían sus sensaciones y opiniones sobre la salud, así como el impacto de la salud en su CVRS. Posteriormente se realizaron 2 grupos. Tras analizar las propiedades psicométricas (fiabilidad y validez) se redujo el número de preguntas y se obtuvo una versión con coeficientes de fiabilidad y validez aceptables ${ }^{12}$. La versión original fue diseñada para uso en encuestas de salud de población general ${ }^{11}$, y ha sido administrado a más de 15.000 adolescentes; en Francia el Ministerio de Educación lo ha recomendado como instrumento de cribado en chequeos médicos en los colegios ${ }^{13}$.
La adaptación de un cuestionario a otra cultura requiere llevar a cabo un proceso sistemático dirigido a obtener una equivalencia semántica y conceptual respecto del original. Existe un cierto consenso respecto a la metodología más adecuada para la adaptación de medidas de salud, denominada traducción directa e inversa o traducción-retrotraducción ${ }^{14-17}$. Una parte fundamental del proceso de adaptación incluye la práctica de técnicas cualitativas (reuniones y discusión en paneles o entrevistas cognitivas) ${ }^{18}$, para garantizar que el contenido del instrumento sea comprensible y que se incorporen aspectos relevantes para la población a la que va dirigido.

Las características propias del VSP-A como medida genérica de CVRS, así como la falta de este tipo de medidas para adolescentes en España, justifican la adaptación del cuestionario. El objetivo del presente estudio fue adaptar el VSP-A para su uso en España, como primera fase para la obtención del cuestionario.

\section{SUJETOS Y MÉTODO}

\section{EI cuestionario VSP-A}

La versión más reciente, que es la adaptada en España, contiene 39 preguntas distribuidas en 11 dimensiones ${ }^{13}$ que son: energía; bienestar psicológico; relaciones con amigos/as; actividades de ocio; relaciones con padres; bienestar físico; relaciones con profesores/as; actividades escolares; autoestima; relaciones con el personal sanitario y satisfacción con la vida sentimental y sexual. Las preguntas se puntúan en una escala Likert de 1 a 5 . A partir de la puntuación media de las preguntas se genera la puntuación de cada dimensión. Por otro lado, el VSP-A permite obtener una puntuación global única de 0 (peor CVRS) a 100 (mejor CVRS) mediante transformaciones lineales de las 11 dimensiones. 


\section{Adaptación de la versión española del VSP-A}

Para la adaptación del cuestionario VSP-A se siguió la metodología de traducción directa e inversa (traducción-retrotraducción) recomendada en la literatura ${ }^{14-17}$. Esta metodo- logía incluyó la traducción al español, la revisión sistemática del contenido del instrumento y discusión por el equipo de investigación, reuniones con grupos de adolescentes, una traducción inversa a la lengua original francesa y administración de la versión pre-test española (figura 1). Los autores ori-

Figura 1

Esquema del proceso de adaptación transcultural del VSP-A

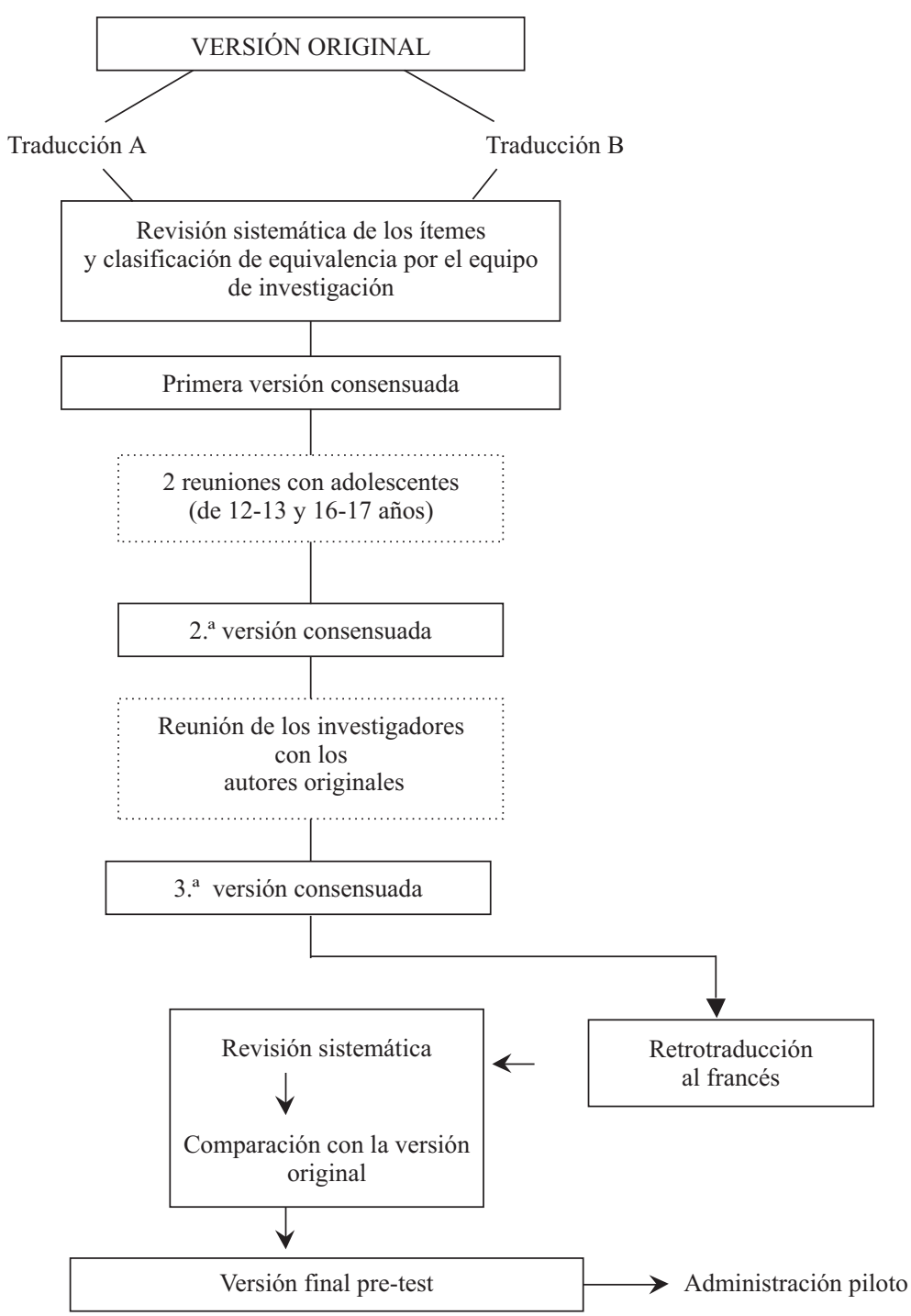


ginales del cuestionario participaron activamente durante todo el proceso de la adaptación a la versión española, mediante reuniones de consenso.

\section{Primera fase: traducción al español}

Dos traductores profesionales de lengua materna española y bilingües en francés tradujeron de forma independiente la versión original francesa del VSP-A al español. El objetivo de esta fase del estudio fue desarrollar una versión semánticamente equivalente a la original, es decir que la versión española transmitiera el significado del original utilizando formulaciones y expresiones de las preguntas que fueran aceptables y culturalmente relevantes para los adolescentes españoles ${ }^{19}$.

El equipo de investigación junto con los traductores revisó de forma sistemática las preguntas del cuestionario, las instrucciones y las opciones de respuesta propuestos por ambos traductores para decidir una primera versión consensuada. Para cuantificar la «adaptabilidad» de su contenido e identificar los problemas iniciales en la adaptación, cada miembro del equipo de investigación puntuó el grado de dificultad en la adaptación inicial de las preguntas en una escala de 0 (dificultad mínima) a 10 (máxima dificultad). A partir de dicha puntuación se clasificaron las preguntas en 3 grupos según los criterios siguientes $^{14}$ :

(A) Preguntas equivalentes: cuando la dificultad en la traducción fue mínima, ambas versiones traducidas fueron similares, y no se plantearon dudas con respecto al vocabulario y el contenido.

(B) Preguntas con problemas en algunas palabras: cuando ambas versiones traducidas presentaban diferencias entre sí, o el equipo de investigación identificó problemas en alguna/s palabra/s, que incluyeron la necesidad de recoger vocablos o expresiones más adecuadas y naturales en español (B1), o bien incorporar aspectos culturales para conseguir una equivalencia semántica y cultural (por ejemplo, se incluyeron algunas actividades de ocio consideradas más adecuadas para los adolescentes en nuestro medio) (B2).

(C) Preguntas sin equivalencia: se definieron como tal cuando el equipo de investigación clasificó la pregunta como inadecuada en nuestro medio.

A partir de las 2 traducciones en español y de los comentarios del equipo español de investigación, se obtuvo la primera versión consensuada del VSP-A.

\section{Segunda fase: reunión y discusión en paneles de adolescentes y consenso del equipo de investigación}

Se realizaron 2 reuniones con adolescentes procedentes de un colegio de titularidad concertada situado en un barrio de nivel socioeconómico bajo. Cada grupo incluyó 8 adolescentes ( 4 chicos y 4 chicas) de edades diferentes: 12-13 años y 16-17 años respectivamente. Los objetivos de esta fase fueron valorar la comprensión de la primera versión consensuada, determinar si el vocabulario era adecuado, y evaluar si las preguntas eran comprensibles y relevantes en nuestra cultura. Las sesiones fueron grabadas y transcritas para llevar a cabo un análisis del contenido de las mismas. Cada sesión tuvo una duración aproximada de una hora. Una persona del equipo de investigación moderaba la sesión y otra recogía notas. Se obtuvo el consentimiento previo de los padres para la participación de sus hijos/as. En cada sesión, los adolescentes cumplimentaron el VSP-A y a continuación se realizaron comentarios en grupo al cuestionario, siguiendo un guión semi-estructurado para comprobar la comprensión de las instrucciones, así como de las preguntas y de las opciones de respuesta, del grado de naturalidad de las expresiones y la aplicabilidad del contenido del cuestionario en nuestro medio. Se trataba de recoger las expresiones de uso común, 
los comentarios y los valores relevantes para los adolescentes. Además se recogieron la edad, el género, la ciudad de nacimiento y la ocupación del padre y de la madre, así como la hora de inicio y de finalización del cuestionario.

Las alternativas propuestas y los comentarios de los adolescentes fueron valoradas posteriormente por el grupo de investigación para determinar dónde se deberían introducir cambios en la primera versión consensuada. La introducción de estos cambios llevó a la segunda versión consensuada del VSP-A en español. Tras una reunión del equipo de investigación con los autores originales del instrumento para discutir algunas dudas respecto al contenido de las preguntas y la equivalencia de la última versión consensuada en español, se obtuvo la tercera versión consensuada del cuestionario.

\section{Tercera fase: retro-traducción al idioma original}

De la tercera versión consensuada del español al francés se realizó una retro-traducción o traducción inversa ${ }^{20}$ por un traductor de lengua materna francesa y bilingüe en español. Los autores originales compararon esta versión retrotraducida con la versión original, con la finalidad de detectar diferencias o discrepancias entre ambas y proponer nuevas formulaciones de las preguntas en español en una reunión de consenso con el equipo de investigación. Estas revisiones de la versión española dieron como resultado la versión pre-test.

\section{Cuarta fase: factibilidad de la administración y comprensión de la versión pre-test}

La versión pre-test se administró en dos grupos de adolescentes de un colegio público de nivel socioeconómico medio de la ciudad de Barcelona: uno de 12-13 años (4 chicos y 4 chicas) y otro de 16-17 años (4 chi- cos y 4 chicas). En esta fase del estudio se valoró la comprensión del vocabulario y la factibilidad de la administración del cuestionario.

\section{RESULTADOS}

El rango de puntuaciones de la dificultad en la adaptación osciló entre 0 y 5,5. Se clasificaron 24 de las 39 preguntas del VSP-A como preguntas (A), 14 como preguntas (B) y 1 pregunta como (C). En la tabla 1 se describen las preguntas de tipo B y C. Las preguntas del cuestionario con mayor dificultad de adaptación en la primera fase fueron: "¿te has sentido rodeado/a, ayudado/a por tus amigos/as? (avez-vous été: entouré(e), aidé(e) par vos copains, vos copines?)»; "¿te has sentido comprendido/a, tranquilizado/a por tu amigos/as? (avez-vous été: compris(e), rassuré(e) par vos copains, vos copines?)»; "¿has tenido dolor en alguna parte de tu cuerpo? (avez-vous pu: des douleurs, mal quelque part?)».

En las tablas 2 y 3 se muestran ejemplos de los comentarios de los adolescentes, incluyendo los de 12-13 y de 16-17 años en relación a las preguntas formuladas en la primera versión consensuada. Por ejemplo, la expresión «agobiado/a» reflejaba de manera más adecuada cómo se sentían respecto al estrés que «estresado/a», que fue considerara una expresión de personas mayores por el grupo de 16-17 años. La palabra «angustiado/a» no era la más adecuada para expresar cómo se sentían con respecto a su futuro y preferían la palabra «preocupado/a». Se sentían «apoyados por los amigos» en lugar de «rodeados».

En relación a la pregunta: «¿te has sentido satisfecho con tu vida sexual?», los participantes de 16-17 años expresaron que, aunque hablar de sexo... «es algo privado.... refleja si te sientes más contento o más triste..» «y es necesario incluirlo en este tipo encuestas». Los más jóvenes en cambio (sobre todo las chicas), consideraron que «te- 
Tabla 1

Clasificación de equivalencia semántica y cultural de preguntas de la $1 .^{\text {a }}$ versión consensuada en español

\begin{tabular}{|c|c|c|c|}
\hline Pregunta versión francesa & Pregunta de la $1 .{ }^{a}$ versión consensuada & $\begin{array}{l}\text { Puntuación } \\
\text { mediana de la } \\
\text { dificultad en } \\
\text { la traducción }\end{array}$ & Equivalencia \\
\hline $\begin{array}{l}\text { Avez-vous été angoissé (e) ou eu peur en pen- } \\
\text { sant à l'avenir? }\end{array}$ & $\begin{array}{l}\text { ¿te has sentido angustiado/a o asustado ante el } \\
\text { futuro? }\end{array}$ & 4 & (B1) \\
\hline $\begin{array}{l}\text { Avez-vous été entouré (e), aidé (e) par vos co- } \\
\text { pains, vos copines? }\end{array}$ & $\begin{array}{l}\text { ¿te has sentido rodeado/a, ayudado/a por tus } \\
\text { amigos/as? }\end{array}$ & 4 & (B1) \\
\hline $\begin{array}{l}\text { Avez-vous compris(e), rassuré(e) par vos co- } \\
\text { pains, vos copines? }\end{array}$ & $\begin{array}{l}\text { ¿te has sentido comprendido/a, tranquilizado/a } \\
\text { por tus amigos/as? }\end{array}$ & 5,5 & (B1) \\
\hline Avez-vous eu: des douleurs, mal quelque part? & ¿has tenido dolor en alguna parte de tu cuerpo? & 3,5 & (B1) \\
\hline $\begin{array}{l}\text { Êtes-vous allé (e) chez vos copains, vos copi- } \\
\text { nes? }\end{array}$ & ¿has ido a casa de tus amigos o amigas? & 4 & (B1) \\
\hline Avez-vous eu le moral? & ¿has tenido la moral alta? & 5 & (B1) \\
\hline $\begin{array}{l}\text { Êtes-vous allé(e) jouer dehors avec vos co- } \\
\text { pains, vos copines (vélo, foot, roller...)? }\end{array}$ & $\begin{array}{l}\text { ¿has hecho actividades al aire libre con tus } \\
\text { amigos o amigas (ir en bici, skating, juegos de } \\
\text { pelota...? }\end{array}$ & 5 & (B2) \\
\hline $\begin{array}{l}\text { Avez-vous pu: sortir (aller en ville, faire des } \\
\text { achats, aller à la piscine, au cinéma...)? }\end{array}$ & $\begin{array}{l}\text { ¿has podido salir (ir al centro, de compras, a la } \\
\text { piscina, al cine...)? }\end{array}$ & 3,5 & (B2) \\
\hline $\begin{array}{l}\text { Avez-vous été entouré(e), aidé(e) par le per- } \\
\text { sonnel soignant (médecin, infirmière, psycho- } \\
\text { logue? }\end{array}$ & $\begin{array}{l}\text { ¿te has sentido rodeado/a, ayudado/a por el } \\
\text { personal sanitario (médico/a, enfermero/a, psi- } \\
\text { cólogo/a)? }\end{array}$ & 4,5 & (B2) \\
\hline $\begin{array}{l}\text { Avez-vous été satisfait(e) de vostre vie sexua- } \\
\text { lle? }\end{array}$ & ¿te has sentido satisfecho de tu vida sexual? & 4 & (C) \\
\hline
\end{tabular}

(A) Preguntas equivalentes

(B) Preguntas con problemas en algunas palabras

B1: Necesidad de incorporar expresiones coloquiales o palabras comprensibles

B2: Necesidad de incorporar aspectos culturales

(C) Preguntas sin equivalencia

nían vida sentimental, no sexual, y que es «una pregunta para mayores».

En la fase de traducción inversa se encontraron 2 discrepancias entre la versión original y la retro-traducción al francés: avez-vous éte: stressé(e)?; entouré(e), aidé(e) par vos copains, vos copines? Por este motivo, se decidió añadir respectivamente, «estresado/a» (de la primera versión consensuada) al término «agobiado/a»; y «ayudado/a» al término «apoyado/a» para mejorar la equivalencia con la versión original.

Finalmente, después de la administración de la versión pre-test a 2 grupos de adolescentes se modificaron los enunciados de 2 preguntas: se añadió «... o han ido ellos a la tuya?» a la pregunta «¿has ido a casa de tus amigos o amigas?»; y se modificó « ¿has tenido dolores?» por la opción propuesta en la
1. ${ }^{\text {a }}$ versión consensuada « ¿has tenido dolor en alguna parte de tu cuerpo?», ya que ésta última formulación presentó menos problemas de comprensión y recogía mejor el dolor físico.

En el anexo se presenta la versión española pre-test del VSP-A.

\section{DISCUSIÓN}

El presente estudio ha permitido obtener la versión española del cuestionario genérico de CVRS para adolescentes VSP-A y constituye la primera fase para la obtención del instrumento de medida. Esta metodología, con algunas variantes ${ }^{14-17}$, se ha utilizado en España para la adaptación de diversos instrumentos de CVRS para adultos y adolescentes ${ }^{21-26}$. La adaptación transcultural de 
Tabla 2

Resultados de los comentarios de los adolescentes sobre la claridad del lenguaje y uso de expresiones cotidianas

\begin{tabular}{|c|c|c|}
\hline $\begin{array}{l}\text { Ejemplos de preguntas de la } 1 .{ }^{\text {a }} \text { versión } \\
\text { consensuada }\end{array}$ & Grupo de adolescentes de $12-13$ años & Grupo de adolescentes de 16-17 años \\
\hline ¿Te has sentido estresado/a? & $\begin{array}{l}\text { «Estresado es lo mismo que desanimado, } \\
\text { cansado». «No tienes tiempo para hacerlo } \\
\text { todo...». }\end{array}$ & $\begin{array}{l}\text { «Estresado es una palabra para más mayo- } \\
\text { res». Prefieren «agobiado» porque es una } \\
\text { palabra más cotidiana. }\end{array}$ \\
\hline $\begin{array}{l}\text { ¿Te has sentido rodeado/a ayudado, por } \\
\text { tus amigos/as? }\end{array}$ & $\begin{array}{l}\text {... «los amigos/as te apoyan, te ayudan». } \\
\text { «Ayudar es que te resuelvan el proble- } \\
\text { ma...». Hay dos ideas en una sola frase. }\end{array}$ & $\begin{array}{l}\text { «Son los padres los que ayudan». «Los } \\
\text { amigos/as te apoyan...». Prefieren apoya- } \\
\text { dos, respaldados que rodeados. "Apoya- } \\
\text { dos por los amigos y ayudados por los pa- } \\
\text { dres...». }\end{array}$ \\
\hline $\begin{array}{l}\text { ¿Has tenido dolor en alguna parte de tu } \\
\text { cuerpo? }\end{array}$ & $\begin{array}{l}\text { «dolor en la barriga...». Los chicos dicen } \\
\text { que han tenido dolor «en el tobillo por el } \\
\text { fútbol, por hacer deporte...». }\end{array}$ & $\begin{array}{l}\text { La alternativa que proponen es: «has teni- } \\
\text { do algún dolor en cualquier parte de tu } \\
\text { cuerpo...». }\end{array}$ \\
\hline $\begin{array}{l}\text { ¿Te has sentido rodeado/a, ayudado/a } \\
\text { por el personal sanitario (médico/a, en- } \\
\text { fermero/a, psicólogo/a? }\end{array}$ & $\begin{array}{l}\text { A diferencia de los amigos/as se sienten } \\
\text { «ayudados/as por el personal sanitario...», } \\
\text { no «rodeados/as, ni apoyados...». }\end{array}$ & $\begin{array}{l}\text {... «más que ayudados se sienten compren- } \\
\text { didos/as, aunque depende del caso...». }\end{array}$ \\
\hline
\end{tabular}

Tabla 3

Resultados de los comentarios de los adolescentes sobre la adecuación cultural del contenido de las preguntas

\begin{tabular}{|c|c|c|}
\hline $\begin{array}{l}\text { Ejemplos de preguntas de la } 1 .^{\text {a }} \text { versión } \\
\text { consensuada }\end{array}$ & Grupo de adolescentes de $12-13$ años & Grupo de adolescentes de 16-17 años \\
\hline $\begin{array}{l}\text { ¿Te has sentido angustiado/a o asustado/a } \\
\text { ante el futuro? }\end{array}$ & $\begin{array}{l}\text {... «angustiado suena muy fuerte». «La } \\
\text { palabra asustado/a equivale a estar preo- } \\
\text { cupado. Mejor poner que te preocupa». } \\
\text { «Le das vueltas a las cosas; piensas en } \\
\text { que las cosas pueden salir mal...». «No es } \\
\text { igual que angustiado». }\end{array}$ & $\begin{array}{l}\text { Más que angustiados que suena muy fuer- } \\
\text { te, se sienten «preocupados...por las no- } \\
\text { tas, la escuela». «Asustados no estamos, } \\
\text { más bien preocupados...» Hacen referen- } \\
\text { cia a preocupaciones escolares, y al futu- } \\
\text { ro inmediato. }\end{array}$ \\
\hline $\begin{array}{l}\text { ¿Has hecho actividades al aire libre con } \\
\text { tus amigos o amigas (ir en bici, skating, } \\
\text { practicar algún deporte...? }\end{array}$ & $\begin{array}{l}\text { Proponen actividades como «pasear, dar } \\
\text { una vuelta, ir en bici...». Una chica dice } \\
\text { «ir al cine», pero el grupo comenta que se } \\
\text { trata de actividades al aire libre. }\end{array}$ & $\begin{array}{l}\text { Una chica comenta que estas actividades } \\
\text { le recuerdan más al verano. «Ir en bici se } \\
\text { puede incluir, andar, pasear, sacar al pe- } \\
\text { rro, deportes (monopatín, fútbol)...». }\end{array}$ \\
\hline $\begin{array}{l}\text { ¿Te has sentido contento/a con tu vida se- } \\
\text { xual? }\end{array}$ & $\begin{array}{l}\text { Se ríen. Les hace gracia la pregunta. Tie- } \\
\text { nen vida sentimental, no sexual y creen } \\
\text { que es una pregunta para mayores. }\end{array}$ & $\begin{array}{l}\text { Se ríen de la expresión. «El sexo es } \\
\text { tabú...son cosas privadas...». «Aunque es } \\
\text { importante hacer preguntas así, pondría } \\
\text { satisfecho de tu vida sexual porque el } \\
\text { sexo te hace gozar...». «Es importante ha- } \\
\text { cer este tipo de pregunta porque te pueden } \\
\text { hacer sentir más contento o más triste...». }\end{array}$ \\
\hline
\end{tabular}

cuestionarios permite obtener instrumentos de medida de CVRS comparables a nivel internacional, realizar estudios multicéntricos que incluyan diferentes países, así como aprovechar la experiencia previa obtenida con el cuestionario. Asimismo, es importante seguir una metodología rigurosa y sistemática para garantizar que las cualidades del instrumento de medida que ha sido adaptado sean similares a las del instrumen- to original (fiabilidad, validez y sensibilidad al cambio), así como para facilitar la aplicabilidad del instrumento en la nueva cultur $\mathrm{ra}^{27,28}$.

Durante la adaptación del VSP-A al español no se encontraron problemas importantes de traducción ni de adaptación del contenido de las preguntas y la puntuación de la equivalencia en la primera fase del proceso 
indicó que las traducciones eran similares al contenido de las preguntas originales. La formulación sencilla de la mayoría de las preguntas así como el hecho de que el cuestionario utiliza pocas expresiones coloquiales en la versión original francesa facilitó su traducción.

Por otra parte, a pesar de las diferencias en el idioma, no se esperaban encontrar diferencias culturales importantes, dada la proximidad geográfica y la pertenencia de ambas poblaciones de adolescentes, tanto la de la versión original como la de la población diana, a culturas mediterráneas.

En el proceso de adaptación de un cuestionario a una nueva cultura es importante realizar reuniones con la población a la cual va dirigido para conocer los significados que dan, en este caso los adolescentes, a los contenidos del cuestionario. Las reuniones con adolescentes permitió redefinir las preguntas con más problemas de adaptación y comprobar la relevancia y la comprensión de los contenidos del cuestionario, como por ejemplo las preguntas sobre la imagen corporal o sobre la satisfacción con la vida sexual.

En general, la comprensión del cuestionario fue buena aunque se propusieron algunos cambios para mejorar la claridad del lenguaje e incorporar expresiones más relevantes para adolescentes. Las preguntas referidas a la apariencia física generaron una reacción de rechazo inicial en algunas de las chicas, aunque reconocieron la importancia del tema para ellas y la necesidad de recoger la autopercepción respecto de la misma. Sólo una pregunta se consideró problemática inicialmente para los adolescentes más jóvenes. Ésta recogía información sobre la satisfacción del adolescente con su vida sexual. Tras los comentarios de los adolescentes, se decidió dejar esta pregunta ya que la mayoría de adolescentes confirmó que podía tener vida sentimental y sexual, aunque algunos de los más jóvenes y algunas chicas comentaron que no tenían «novio/a» ni vida sexual. Por este motivo se decidió incluir en la segunda versión consensuada la opción de respuesta «no tengo» en las preguntas sobre satisfacción del adolescente con su vida sentimental y sexual, así como en las preguntas referidas al contacto con el personal sanitario «no me han visitado», para aquellos adolescentes que no tenían vida sentimental/sexual o que no habían tenido contacto con el personal sanitario durante las 4 últimas semanas.

La introducción de esta opción de respuesta para las preguntas relacionadas con la vida sexual y en las preguntas sobre uso de servicios sanitarios es la principal diferencia de la versión adaptada respecto del original y merece ser comentada. De hecho, en el cuestionario original existe una opción de respuesta parecida [«non concerné(e)», «no me afecta»] en todas las preguntas. Sin embargo, se decidió eliminarla después de extensas discusiones del equipo investigador debido a los importantes problemas de comprensión detectados en las reuniones con adolescentes. En muchas ocasiones los adolescentes inicialmente seleccionaron la opción «no me afecta» para indicar que no les pasaba nada (por ejemplo, no se habían sentido tristes en las últimas 4 semanas). Por este motivo, se decidió incluir sólo las alternativas de respuesta para especificar que no tenían vida sexual o sentimental o que no habían utilizado los servicios sanitarios en las últimas 4 semanas.

Finalmente, la participación de los autores originales en todo el proceso ha sido importante para obtener una versión adecuada y equivalente. Tras la retro-traducción de la $3 .^{\text {a }}$ versión consensuda al francés, en la que los autores originales confirmaron que el proceso de adaptación de la versión española había mantenido el contenido y significado de cada una de las preguntas de la versión original, se produjeron muy pocos cambios, probablemente porque el proceso anterior había sido riguroso y sistemático. 
Una limitación del estudio fue que en ningún momento se intentó averiguar con preguntas abiertas el concepto que los adolescentes tienen de la calidad de vida, porque se trataba de la adaptación de un cuestionario ya elaborado. Por tanto, es posible que algunos temas relevantes para adolescentes en España no se hayan incorporado; sin embargo, esto es una limitación de este tipo de proceso de adaptación y sería interesante llevar a cabo estudios futuros que intentasen investigar en más profundidad la opinión de los adolescentes en España respecto a su concepto de la calidad de vida.

Por otra parte, el hecho de obtener una versión del VSP-A adaptada al español no significa que el instrumento tenga las mismas propiedades psicométricas que la versión original. En futuros estudios será necesario evaluar la fiabilidad (consistencia interna y estabilidad test-retest) y la validez del instrumento (constructo, convergente/discriminante, criterio y estructural), así como su sensibilidad al cambio ${ }^{27,28}$. También sería conveniente probar la factibilidad de la administración del cuestionario en varias regiones, así como adaptar versiones que incluyan otras lenguas oficiales para uso en comunidades como Cataluña o País Vasco.

Una vez comprobadas sus propiedades psicométricas, el VSP-A será un cuestionario de CVRS útil, sencillo y corto, con un tiempo de administración de 10-15 minutos, para uso en la práctica diaria y en diversos ámbitos de la salud pública. El VSP-A podrá ser utilizado en encuestas de salud o como instrumento de cribado para detectar diferencias sistemáticas entre grupos de adolescentes con diversas características (sociodemográficas o clínicas) y relacionarlas con potenciales diferencias en el estado de salud y en el acceso a los servicios sanitarios ${ }^{11}$. El instrumento también podrá ser útil para establecer necesidades en salud y plantear estrategias para mejorar la CVRS de los adolescentes.

\section{AGRADECIMIENTOS}

Las/os autores agradecen la participación de los colegios de enseñanza secundaria IES Sant Francesc e IES Les Corts, así como la participación de los/as adolescentes en este estudio.

\section{BIBLIOGRAFÍA}

1. Fayers MP, Machin D. Quality of life: assessment, analysis and interpretation. Chichester: John Wiley Sons; 2000.

2. Hennessy CH, Moriarty DG, Zach MM, Scherr P, Brackbill R. Measuring health-related quality of life for public health surveillance. Public Health Rep 1994; 109:665-772.

3. Kind P. Measuring quality of life in evaluating clinical interventions: an overview. Ann Med 2001; 33:323-7.

4. Alonso J. La medida de la calidad de vida relacionada con la salud en la investigación y en la práctica clínica. Gac Sanit 2000; 14:163-67.

5. Pinto-Prades JL. Calidad de vida y asignación de recursos sanitarios. Gac Sanit 2000; 14:168-74.

6. Guyatt GH, Feeny DH, Patrick DL. Measuring health-related quality of life. Ann Int Med 1993; 118:622-9.

7. Shumaker S, Naughton M. The International assessment of health-related quality of life: a theoretical perspective. En: Shumaker S, Berton R editors. The International assessment of health-related quality of life: theory, translation, measurement and analysis. Oxford: Rapid Communications; 1995.

8. Eiser C, Morse R. A review of measures of quality of life for children with chronic illness. Arch Dis Child 2001; 84:205-211.

9. Rajmil L, Estrada MD, Herdman M, Serra-Sutton $\mathrm{V}$, Alonso J. Calidad de vida relacionada con la salud (CVRS) en la infancia y en la adolescencia: revisión de la literatura y de los instrumentos adaptados en España. Gac Sanit 2001; 15:34-43.

10. Siméoni MC, Auquier P, Antoniotte S, Sapin C, San Marco JL. Validation of a French health-related quality of life intrument for adolescents: The VSP-A. Qual Life Res 2000; 9:393-403. 
11. Siméoni MC, Sapin C, Antoniotti S, Auquier P Health-related quality of life reported by French adolescents: a predictive approach of health status? J Adolesc Health 2001; 28:288-94.

12. Siméoni MC, Spain C, Antoniotti S, Michel G, Auquier P. Validation of the VSP-AM: A French HRQL questionnaire for ill adolescents. Qual Life Res 2000; 9:298.

13. Siméoni MC, Sapin C, Clement A, El Khammar M, Auquier P. The core VSP-A and its specific module: a generic HRQL instrument for ill and healthy adolescents. Qual Life Res 2001; 10:267.

14. Alonso J, Antó JM. Instrumentos de medida de calidad de vida relacionada con la salud: características generales y proceso de adaptación transcultural. Quaderns CAPS 1990; 14: 16-24.

15. Guyatt GH. The philosophy of health - related quality of life translation. Qual Life Res 1993; $2: 461-5$.

16. Bullinger M, Alonso J, Apolone G, Leplège A, Sullivan M. et al. Translating health status questionnaires and evaluating their quality: the IQOLA approach. J Clin Epidemiol 1998; 51:913-23.

17. Beaton DE, Bombardier C, Guillemin F, Bosi-Ferraz M. Guidelines for the process of cross-cultural adaptation of self-report measures. Spine 2000; 25:3186-91

18. Walker R. Applied qualitative research. Aldershot: Gower; 1985.

19. Herdman M, Fox-Rushby, Badia X. A model of equivalence in the cultural adaptation of HRQoL instruments: the universalist approach. Qual Life Res 1998; 7:323-335.

20. Guillemin F, Bombardier C, Beaton D. Cross-cultural adaptation of health-related quality of life measures: literature review and proposed guidelines. J Clin Epidemiol 1993;
46:1417-32.

21. Alonso J, Antó JM, Moreno C. Spanish version of the Nottingham health profile: translation and preliminary validity. AJPH 1990; 80:704-8.

22. Ferrer M, Alonso J, Prieto L, Plaza V, Monso E, Marrades R, et al. Validity and reliability of the St. George's Respiratory Questionnaire after adaptation to a different language and culture: the Spanish example. Eur Respir J 1996; 9: 1160-6.

23. Alonso J, Prieto L, Antó JM. La versión española del «SF-36 Health Survey» (Cuestionario de salud SF-36): un instrumento de medida de resultados clínicos. Med Clín (Barc) 1995; 104:771-6.

24. Sanjuàs $\mathrm{C}$, Alonso $\mathrm{J}$, Sanchís $\mathrm{J}$, Casan $\mathrm{P}$, Broquetas JM, Ferrie PJ, Juniper EF, Antó JM. Cuestionario de calidad de vida en pacientes con asma: la versión española del Asthma Quality of Life Questionnaire. Archivos de Bronconeumología 1995; 31: 219-26.

25. Tauler E, Vilagut G, Grau G, González A, Sánchez E, Figueras G, Vall O, Ferrer M, Alonso J. The Spanish version of the Paediatric Asthma Quality of Life Questionnaire (PAQLQ): Metric characteristics and equivalence with the original version. Qual Lif Res 2001; 10:81-91.

26. Rajmil L, Serra-Sutton V, Alonso J, Starfield B, Riley A, Vázquez JR. The Spanish version of the Child Health and Illness Profile, Adolescent Edition (CHIP-AE). Qual Lif Res (en prensa)

27. Hays RD, Anderson R, Revicki D. Psychometric considerations in evaluating health related quality of life measures. Qual Life Res 1993; 2:441-9.

28. Aaronson N, Alonso J, Burnam A, Lohr KN, Patrick DL, Stein R Assessing health status and quality-of-life instruments: Attributes and review criteria. Qual Lif Res 2002; 11:193-205. 


\section{ANEXO}

Versión española del cuestionario VSP-A

Tu salud y calidad de vida

Durante las cuatro últimas semanas,

\begin{tabular}{|lccccc|}
\hline & $\begin{array}{c}\text { Nunca } \\
\text { Nada }\end{array}$ & $\begin{array}{c}\text { Pocas veces } \\
\text { Poco }\end{array}$ & $\begin{array}{c}\text { Algunas veces } \\
\text { Moderada- } \\
\text { mente }\end{array}$ & $\begin{array}{c}\text { Muchas veces } \\
\text { Mucho }\end{array}$ & $\begin{array}{c}\text { Siempre } \\
\text { Muchisimo }\end{array}$ \\
\hline 1. ¿Te has sentido inquieto/a, preocupado/a? & $\square$ & $\square$ & $\square$ & $\square$ & $\square$ \\
2. ¿Te has sentido triste? & $\square$ & $\square$ & $\square$ & $\square$ & $\square$ \\
3. ¿Te has sentido estresado/a, agobiado/a? & $\square$ & $\square$ & $\square$ & $\square$ & $\square$ \\
4. ¿Te has desanimado con facilidad? & $\square$ & $\square$ & $\square$ & $\square$ & $\square$ \\
5. ¿Te has sentido preocupado/a por el futuro? & $\square$ & $\square$ & $\square$ & $\square$ & $\square$ \\
6. ¿Te has sentido satisfecho/a con tu vida? & $\square$ & $\square$ & $\square$ & $\square$ & $\square$ \\
7. ¿Te has sentido ayudado/a, apoyado/a por tus & $\square$ & $\square$ & $\square$ & $\square$ & $\square$ \\
amigos/as? & ¿Te has sentido comprendido/a por tus ami- & $\square$ & $\square$ & $\square$ & $\square$ \\
gos/as? & & $\square$ & $\square$ \\
\hline
\end{tabular}

Durante las cuatro últimas semanas,

\begin{tabular}{|lcccccc|}
\hline & No tengo & $\begin{array}{c}\text { Nunca } \\
\text { Nada }\end{array}$ & $\begin{array}{c}\text { Pocas ve- } \\
\text { ces } \\
\text { Poco }\end{array}$ & $\begin{array}{c}\text { Algunas veces } \\
\text { Modera- } \\
\text { damente }\end{array}$ & $\begin{array}{c}\text { Muchas } \\
\text { veces } \\
\text { Mucho }\end{array}$ & $\begin{array}{c}\text { Siempre } \\
\text { Muchisimo }\end{array}$ \\
\hline $\begin{array}{l}\text { 9. ¿Te has sentido satisfecho/a con la rela- } \\
\text { ción sentimental con tu chico/a? }\end{array}$ & $\square$ & $\square$ & $\square$ & $\square$ & $\square$ & $\square$ \\
$\begin{array}{l}\text { 10. ¿Te has sentido satisfecho/a con tu vida } \\
\text { sexual? }\end{array}$ & $\square$ & $\square$ & $\square$ & $\square$ & $\square$ & $\square$ \\
\hline
\end{tabular}

Durante las cuatro últimas semanas,

\begin{tabular}{|c|c|c|c|c|c|}
\hline & $\begin{array}{l}\text { Nunca } \\
\text { Nada }\end{array}$ & $\begin{array}{c}\text { Pocas veces } \\
\text { Poco }\end{array}$ & $\begin{array}{c}\text { Algunas veces } \\
\text { Moderada- } \\
\text { mente }\end{array}$ & $\begin{array}{c}\text { Muchas veces } \\
\text { Mucho }\end{array}$ & $\begin{array}{l}\text { Siempre } \\
\text { Muchísimo }\end{array}$ \\
\hline $\begin{array}{l}\text { 11. ¿Te has sentido comprendido/a, tranquili- } \\
\text { zado/a por tus padres? }\end{array}$ & $\square$ & $\square$ & $\square$ & 口 & 口 \\
\hline $\begin{array}{l}\text { 12. ¿Te has sentido satisfecho/a de tus resulta- } \\
\text { dos escolares? }\end{array}$ & $\square$ & $\square$ & $\square$ & $\square$ & $\square$ \\
\hline $\begin{array}{l}\text { 13. ¿Te has sentido ayudado/a por tus profeso- } \\
\text { res? }\end{array}$ & $\square$ & $\square$ & $\square$ & $\square$ & $\square$ \\
\hline $\begin{array}{l}\text { 14. ¿Te has sentido comprendido/a por tus } \\
\text { profesores? }\end{array}$ & $\square$ & $\square$ & $\square$ & $\square$ & $\square$ \\
\hline $\begin{array}{l}\text { 15. ¿Te has sentido aceptado/a, respetado/a } \\
\text { por tus profesores? }\end{array}$ & $\square$ & 口 & $\square$ & $\square$ & $\square$ \\
\hline
\end{tabular}


Durante las cuatro últimas semanas,

\begin{tabular}{|c|c|c|c|c|c|c|}
\hline & & $\begin{array}{l}\text { Nunca } \\
\text { Nada }\end{array}$ & $\begin{array}{l}\text { Pocas veces } \\
\text { Poco }\end{array}$ & $\begin{array}{l}\text { Algunas veces } \\
\text { Modera- } \\
\text { damente }\end{array}$ & $\begin{array}{l}\text { Muchas veces } \\
\text { Mucho }\end{array}$ & $\begin{array}{c}\text { Siempre } \\
\text { Muchísimo }\end{array}$ \\
\hline \multicolumn{7}{|c|}{ 16. ¿Te has sentido acomplejado/a por tu físico, por } \\
\hline & $\begin{array}{l}\text { ¿Te has sentido demasiado gordo/a, demasiado } \\
\text { delgado/a, demasiado alto/a o demasiado bajo/a? }\end{array}$ & 口 & 口 & $\square$ & $\square$ & $\square$ \\
\hline & ¿Te ha faltado energía? & $\square$ & $\square$ & $\square$ & $\square$ & $\square$ \\
\hline & ¿Has estado en buena forma física? & $\square$ & $\square$ & $\square$ & $\square$ & $\square$ \\
\hline 20. & ¿Te has sentido débil, cansado/a? & $\square$ & $\square$ & $\square$ & $\square$ & $\square$ \\
\hline & ¿Te han dado tus padres algún consejo? & $\square$ & $\square$ & $\square$ & $\square$ & $\square$ \\
\hline \multicolumn{7}{|c|}{$\begin{array}{l}\text { 22. ¿Has ido a casa de tus amigos o amigas o han ido } \\
\text { ellos/as a la tuya? }\end{array}$} \\
\hline 23. & $\begin{array}{l}\text { ¿Has hecho algún tipo de actividad al aire libre } \\
\text { con tus amigos o amigas: ir en bici, practicar al- } \\
\text { gún deporte...? }\end{array}$ & $\square$ & $\square$ & $\square$ & $\square$ & $\square$ \\
\hline 24. & ¿Te has sentido con la moral alta? & $\square$ & $\square$ & $\square$ & $\square$ & $\square$ \\
\hline 25. & ¿Te has tomado las cosas por su lado positivo? & $\square$ & $\square$ & $\square$ & $\square$ & $\square$ \\
\hline \multicolumn{2}{|r|}{ 26. ¿Has tenido la sensación de que todo iba bien a tu } & $\square$ & $\square$ & $\square$ & $\square$ & ם \\
\hline \multicolumn{6}{|c|}{ 27. ¿Has tenido confianza en ti mismo/a, te has senti- } & ם \\
\hline \multicolumn{2}{|r|}{ 28. ¿Has tenido buenos resultados en el colegio, en el } & $\square$ & $\square$ & $\square$ & $\square$ & $\square$ \\
\hline \multicolumn{2}{|r|}{ 29. ¿Has tenido dolor en alguna parte de tu cuerpo? } & $\square$ & $\square$ & $\square$ & $\square$ & $\square$ \\
\hline \multicolumn{7}{|c|}{ 30. ¿Has podido hablar libremente con tus amigos/as, } \\
\hline \multirow{2}{*}{\multicolumn{2}{|c|}{$\begin{array}{l}\text { 31. ¿Has podido reunirte con tu grupo de amigos/as? } \\
\text { 32. ¿Has podido salir: ir al centro, de compras, al } \\
\text { cine, dar una vuelta...? }\end{array}$}} & $\square$ & $\square$ & $\square$ & $\square$ & $\square$ \\
\hline & & $\square$ & $\square$ & $\square$ & $\square$ & $\square$ \\
\hline \multirow{2}{*}{\multicolumn{2}{|c|}{$\begin{array}{l}\text { 33. ¿Has podido charlar con tus amigos o amigas? } \\
\text { 34. ¿Has podido sincerarte, hablar de tus problemas } \\
\text { con tus amigos/as? }\end{array}$}} & $\square$ & $\square$ & $\square$ & $\square$ & $\square$ \\
\hline & & $\square$ & $\square$ & $\square$ & $\square$ & $\square$ \\
\hline \multicolumn{6}{|c|}{$\begin{array}{l}\text { 35. ¿Has podido sincerarte, hablar de tus problemas } \\
\text { con tus padres? }\end{array}$} & $\square$ \\
\hline & $\begin{array}{l}\text { ¿Has podido hablar libremente con tus padres, dar } \\
\text { tu opinión? }\end{array}$ & $\square$ & $\square$ & $\square$ & $\square$ & ם \\
\hline
\end{tabular}

Durante las cuatro últimas semanas,

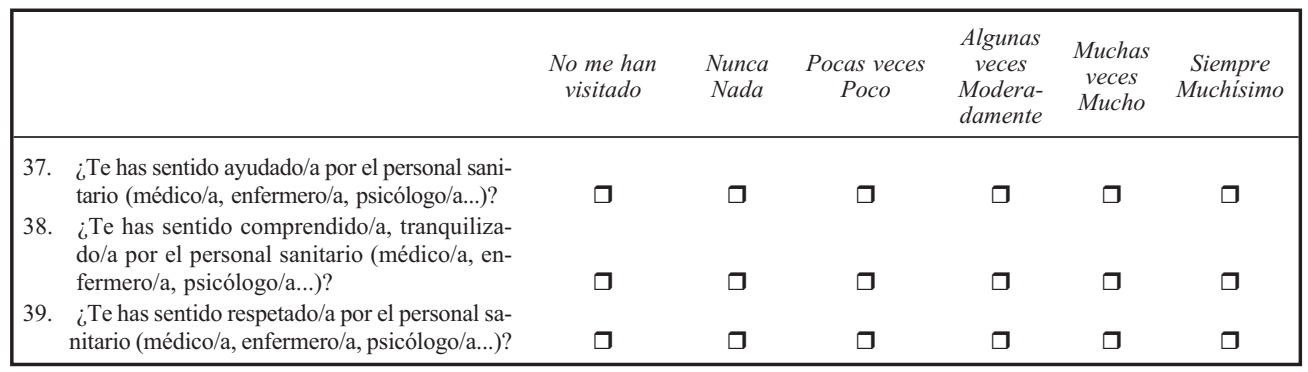

